https://doi.org/10.15407/ujpe64.11.1069

YU.A. SITENKO ${ }^{1}$ V.M. GORKAVENKO ${ }^{2}$

${ }^{1}$ Bogolyubov Institute for Theoretical Physics, National Academy of Sciences of Ukraine

(14-b, Metrolohichna Str., Kyiv 03143, Ukraine)

2 Taras Shevchenko National University of Kyiv, Ukraine

(64, Volodymyrs'ka Str., Kyiv 01601, Ukraine)

\title{
POLARIZATION OF THE VACUUM \\ OF THE QUANTIZED SPINOR FIELD \\ BY A TOPOLOGICAL DEFECT \\ IN THE TWO-DIMENSIONAL SPACE ${ }^{1}$
}

\begin{abstract}
The two-dimensional space with a topological defect is a transverse section of the threedimensional space with an Abrikosov-Nielsen-Olesen vortex, i.e. a gauge-flux-carrying tube which is impenetrable for quantum matter. Charged spinor matter field is quantized in this section with the most general mathematically admissible boundary condition at the edge of the defect. We show that a current and a magnetic field are induced in the vacuum. The dependence of results on the boundary conditions is studied, and we find that the requirement of finiteness of the total induced vacuum magnetic flux removes an ambiguity in the choice of boundary conditions. The differences between the cases of massive and massless spinor matters are discussed.

Ke ywords: vacuum polarization, vortex, current, magnetic flux.
\end{abstract}

\section{Introduction}

Topological phenomena are of great interest and importance because of their universal nature connected with general properties of space-time, on the one hand, and their numerous practical aspects, on the other hand. In 1959, Aharonov and Bohm [1] considered the quantum-mechanical scattering of a charged particle on a magnetic vortex and found an effect that does not depend on the depth of penetration of the charged particle into the region of the vortex flux. Thus, it was demonstrated for the first time that the quantum-mechanical motion of charged particles can be affected by the magnetic flux from the region impenetrable for the particles, if the first homotopy group of the region accessible to the particles is nontrivial. This effect which is alien to classical physics has a great impact on the development of various fields in quantum physics, ranging from particle physics and cosmology to condensed matter and mesoscopic physics (see, e.g., reviews [2-4]). However, even more important is that the discovery made by Aharonov and Bohm revealed a significance of

(C) YU.A. SITENKO, V.M. GORKAVENKO, 2019

ISSN 2071-0194. Ukr. J. Phys. 2019. Vol. 64, No. 11 topology in the context of fundamental principles of quantum theory.

In 1957, Abrikosov [5] discovered that a magnetic vortex can be formed in the type-II superconductors. Later, this result was rederived in a more general context in relativistic field theory [6]. Such string-like structures denoted as the Abrikosov-Nielsen-Olesen (ANO) vortices arise as topological defects in the aftermath of phase transitions with spontaneous breakdown of continuous symmetries; the general condition of the existence of these structures is that the first homotopy group of the group space of the broken symmetry group be nontrivial.

At present, much attention is paid to the study of nonperturbative effects in quantum systems, arizing as a consequence of the interaction of quantized matter fields with a topologically nontrivial classical field background. The ANO vortex is described classically in terms of a spin-0 (Higgs) field which condenses and a spin-1 field corresponding to the spontaneously broken gauge group; the former field is coupled to the

1 This work is based on the results presented at the XI BolyaiGauss-Lobachevskii (BGL-2019) Conference: Non-Euclidean, Noncommutative Geometry and Quantum Physics. 
latter one in the minimal way with constant $\tilde{e}_{\text {cond }}$. Single-valuedness of the condensate field and finiteness of the vortex energy implement that the vortex flux is related to $\tilde{e}_{\text {cond }}$ :

$\Phi=\oint d \mathbf{x} \mathbf{V}(\mathbf{x})=2 \pi / \tilde{e}_{\text {cond }}$

where $\mathbf{V}(\mathbf{x})$ is the vector potential of the spin-1 gauge field, and the integral is over a path enclosing the vortex tube once (natural units $\hbar=c=1$ are used). While considering the effect of the ANO vortex on quantized matter fields, the following circumstances should be taken into account. The quantized matter field couples to a vector potential of the vortex-forming gauge field in the minimal way with coupling constant $\tilde{e}$. The ANO vortex is characterized by two cores: the one (where the gauge symmetry is unbroken) has the transverse size of the order of correlation length, and the other one (where the gauge field strength is nonzero) has the transverse size of the order of penetration depth. The value of the quotient of these length scales is known as the Ginzburg-Landau parameter, and this value distinguishes between superconductors of type-I and type-II (for a review, see, e.g., [7]). Not going into details, we would like to emphasize here that the phase with spontaneously broken symmetry, where the matter field is quantized, exists in a spatial region outside the vortex, and this region is not simply connected: its first homotopy group is nontrivial. Hence, we anticipate in close analogy with the Aharonov-Bohm effect that the ANO vortex has no effect on the surrounding matter in the framework of classical theory, and such an effect, if exists, is of purely quantum nature. Note also that, in view of the above, the issue of boundary conditions for a quantized matter field at the edge of the vortex takes a special significance. The least restrictive, but still physically acceptable, is the condition that ensures the self-adjointness of the Hamiltonian operator (see, e.g., [8]).

The stress-energy tensor corresponding to the ANO vortex has diagonal nonvanishing components only: $-T_{z z}=T_{00}>0,0<-T_{r r} \ll T_{00}, 0<-r^{-2} T_{\varphi \varphi} \ll$ $\ll T_{00}$ (see, e.g., [9]). The stress-energy tensor is a source of gravity according to the Einstein-Hilbert equation

$R_{\rho \rho^{\prime}}-\frac{1}{2} g_{\rho \rho^{\prime}} R=8 \pi \mathrm{G} T_{\rho \rho^{\prime}}$, where $R_{\rho \rho^{\prime}}$ is the Ricci tensor, $R=g^{\rho \rho^{\prime}} R_{\rho \rho^{\prime}}$ is the scalar curvature, and $\mathrm{G}$ is the gravitational constant; we use the notations adopted in [10]. Taking the trace over Lorentz indices in (2), one gets that the spacetime region of the vortex core is characterized by the positive scalar curvature, $R>16 \pi \mathrm{G} T_{00}$, since $T_{00}$ is positive there. The space-time outside the vortex core is flat $(R=0)$, but non-Minkowskian, with the metric given by the squared length element

$d s^{2}=-d t^{2}+d r^{2}+\nu^{-2} r^{2} d \varphi^{2}+d z^{2}$,

where

$\nu=(1-4 \mathrm{G} M)^{-1}$,

$M$ is the linear density of a mass stored in the core, which can be estimated to be of the order of the squared mass of the condensate field. A transverse ( $z=$ const) section of the outer space is a conical surface with the deficit angle equal to $8 \pi \mathrm{G} M$.

Quantum-field-theoretical models in the $(2+1)$ dimensional space-time exhibit a lot of interesting features such as the fermion number fractionization, parity violation, and flavor symmetry breaking (for the review, see $[11,12])$. A regular configuration (i.e. a function continuous in the whole that can grow at most as $O\left(\left|\mathbf{x}-\mathbf{x}_{w}\right|^{-2+\varepsilon}\right)(\varepsilon>0)$ at separate points) of a magnetic field induces the fermion number in the vacuum of a quantized spinor matter field in the two-dimensional space (surface) which is pierced by the magnetic field strength lines; the fermion number density is proportional to the field strength, and the total fermion number is proportional to the total field flux [13]. The effect of a singular configuration of the magnetic field on the vacuum is quite different; the point where the field strength pierces the surface is punctured, and the total vacuum fermion number which is induced on the surface out of a puncture is periodic in the value of the total flux of the singular field configuration. This was realized in a rather general context in [14-16], where it was proven for the first time that the flux through the regions nonaccessible for the quantized spinor matter field induces the fermion number in its vacuum, thus providing a manifestation of the Aharonov-Bohm effect (that is characterized by the periodic dependence on the excluded magnetic flux) [1] in quantum field theory.

The case of the excluded magnetic flux is similar to the case of a topological defect in the form of the ANO vortex. In the last case, the role of a magnetic

ISSN 2071-0194. Ukr. J. Phys. 2019. Vol. 64, No. 11 
field is played by the gauge field corresponding to the spontaneously broken symmetry, and the vacuum of a quantized spinor matter field exists out of the vortex core. As a first step, one can neglect the transverse size of the vortex and formally put the correlation length equal to zero. However, the issue of the choice of boundary conditions even for a vortex with the vanishing transverse size is of primary importance. This issue was not touched upon in [14-16], it was elaborated later with the use of the most general set of boundary conditions ensuring the self-adjointness of the relevant Dirac Hamiltonian operator. Namely, all vacuum polarization effects which are induced by a singular vortex in quantum spinor matter were obtained in [17-21] for the case of massive spinor and in [22-24] for the case of massless spinor. It should be noted that some vacuum polarization effects in the background of a singular vortex were considered earlier in [25-27] for particular boundary conditions; however, the results in $[26,27]$ are actually erroneous, since the periodicity in the value of the flux of a singular vortex was overlooked in an endeavor to imitate the results which are appropriate for the case of regular field configuration.

As a next step, one has to take the conicity of space out of the vortex into account. This task was considered in a number of papers, see [28-35], for quantized both scalar and spinor matter fields, sometimes incompletely and inconclusively as regards to the case of massive matter. The last step is to take the nonvanishing correlation length into account, i.e. the transverse size of a vortex. This task was considered in [3638 ] for quantum spinor matter under a specific boundary condition, and in [39-42] for quantum scalar matter under the Dirichlet boundary condition in space of arbitrary dimension.

The aim of the present work is to study the impact of a boundary condition of the most general form on the vacuum polarization effects which are induced by the ANO vortex in quantum spinor matter in $(2+1)$ dimensional space-time. Of primary interest are such characteristics of the vacuum, as current, parityviolating condensate and energy-momentum tensor ${ }^{2}$, since the fermion number and angular momentum

\footnotetext{
2 It should be noted that current and energy-momentum tensor are the only vacuum characteristics which are induced by the ANO vortex in quantum scalar matter in space-time of arbitrary dimension, see [43-45].
}

change sign under the transition to the inequivalent irreducible representation of the Dirac-Clifford algebra in $(2+1)$-dimensional space-time.

\section{Current and Magnetic Field Which are Induced in the Vacuum}

Postponing the consideration of parity-violating condensate and energy-momentum tensor to subsequent publications, we start with the induced vacuum current which is given by expression

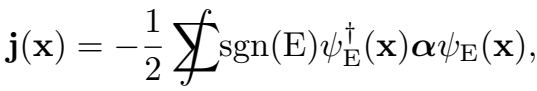

where $\psi_{E}(\mathbf{x})$ is the solution to the stationary Dirac equation,

$$
\begin{aligned}
& H \psi_{E}(\mathbf{x})=E \psi_{E}(\mathbf{x}) \\
& H=-\mathrm{i} \boldsymbol{\alpha}\left(\boldsymbol{\partial}-\mathrm{i} \tilde{e} \mathbf{V}+\frac{\mathrm{i}}{2} \boldsymbol{\omega}\right)+\beta m
\end{aligned}
$$

$\mathbf{V}(\mathbf{x})$ and $\boldsymbol{\omega}(\mathbf{x})$ are the bundle and spin connections, symbol $\&$ denotes the summation over the discrete part and the integration over the continuous part of the energy spectrum, and $\operatorname{sgn}(u)$ is the sign function $(\operatorname{sgn}(\mathrm{u})= \pm 1$ at $u \gtrless 0)$. As a consequence of the Maxwell equation,

$\boldsymbol{\partial} \times \mathbf{B}_{\mathrm{I}}(\mathbf{x})=e \mathbf{j}(\mathbf{x})$,

the magnetic field strength, $\mathbf{B}_{\mathrm{I}}(\mathbf{x})$, is also induced in the vacuum; here, the electromagnetic coupling constant, $e$, differs in general from $\tilde{e}$. The total flux of the induced vacuum magnetic field is

$\Phi_{\mathrm{I}}=\int d \boldsymbol{\sigma} \mathbf{B}_{\mathrm{I}}(\mathbf{x})$

In the background of the ANO vortex, the only one component of the bundle and spin connections is nonvanishing:

$V_{\varphi}=\frac{\Phi}{2 \pi}, \quad w_{\varphi}=\mathrm{i} \frac{\nu-1}{r} \alpha_{\varphi} \alpha_{r}$

and the Dirac Hamiltonian operator takes the form

$H=-\mathrm{i}\left[\alpha^{r}\left(\partial_{r}+\frac{1-\nu}{2 r}\right)+\alpha^{\varphi}\left(\partial_{\varphi}-\mathrm{i} \frac{\tilde{e} \Phi}{2 \pi}\right)\right]+\beta m$, 
where

$$
\begin{aligned}
& \alpha^{r}=\alpha_{r}=\left(\begin{array}{cc}
0 & \mathrm{i} e^{-\mathrm{i} \varphi} \\
-\mathrm{i} e^{\mathrm{i} \varphi} & 0
\end{array}\right), \\
& \alpha^{\varphi}=\frac{\nu}{r}\left(\begin{array}{cc}
0 & e^{-\mathrm{i} \varphi} \\
e^{\mathrm{i} \varphi} & 0
\end{array}\right), \quad \alpha_{\varphi}=\frac{r^{2}}{\nu^{2}} \alpha^{\varphi} .
\end{aligned}
$$

Decomposing the function $\psi_{E}(\mathbf{x})$ as

$\psi_{E}(\mathbf{x})=\sum_{n \in \mathbb{Z}}\left(\begin{array}{c}f_{n}(r, E) e^{\mathrm{i} n \varphi} \\ g_{n}(r, E) e^{\mathrm{i}(n+1) \varphi}\end{array}\right)$

( $\mathbb{Z}$ is the set of integer numbers), we present the Dirac equation as a system of two first-order differential equations for radial functions:

$$
\begin{aligned}
& {\left[-\partial_{r}+\frac{\nu\left(n-n_{\mathrm{c}}-F+\frac{1}{2}\right)-\frac{1}{2}}{r}\right] f_{n}=(E+m) g_{n},} \\
& {\left[\partial_{r}+\frac{\nu\left(n-n_{\mathrm{c}}-F+\frac{1}{2}\right)+\frac{1}{2}}{r}\right] g_{n}=(E-m) f_{n},}
\end{aligned}
$$

where

$\left.n_{\mathrm{c}}=\llbracket\left|\frac{\tilde{e} \Phi}{2 \pi}\right|\right], \quad F=\left\{\left|\frac{\tilde{e} \Phi}{2 \pi}\right|\right\}$,

$[|u|]$ is the integer part of the quantity $u$ (i.e. the integer which is less than or equal to $u$ ), and $\{|u|\}=$ $=u-[|u|]$ is the fractional part of the quantity $u, 0 \leq\{|u|\}<1$. Using (11) and (12), one gets $j_{r}=0$, and the only component of the induced vacuum current,

$j_{\varphi}(r)=-\frac{r}{\nu} \bigvee \sum_{n \in \mathbb{Z}} \operatorname{sgn}(E) f_{n}(r, E) g_{n}(r, E)$,

is independent of the angular variable. The induced vacuum magnetic field strength is directed along the vortex axis,

$B_{\mathrm{I}}(r)=e \nu \int_{r}^{\infty} \frac{d r^{\prime}}{r^{\prime}} j_{\varphi}\left(r^{\prime}\right)$,

with the total flux

$\Phi_{\mathrm{I}}=\frac{2 \pi}{\nu} \int_{r_{0}}^{\infty} d r r B_{\mathrm{I}}(r)$,

where it is assumed without loss of generality that the vortex core has the form of a tube of radius $r_{0}$.

1072
We prove that the most general boundary condition ensuring the self-adjointness of the operator $H(10)$ is

$\left.\left(I-\mathrm{i} \beta \alpha^{r} e^{-\mathrm{i} \theta \alpha^{r}}\right) \psi\right|_{r=r_{0}}=0$,

where $\theta$ is the self-adjoint extension parameter. This condition is also the most general one ensuring the absence of the matter flux across the vortex core edge, i.e. the confinement of the matter field to the region out of the vortex core. Imposing the boundary condition (18) on the solution to the Dirac equation, $\psi_{E}(\mathbf{x})$ (12), we obtain the condition for the modes:

$\cos \left(\frac{\theta}{2}+\frac{\pi}{4}\right) f_{n}\left(r_{0}, E\right)=-\sin \left(\frac{\theta}{2}+\frac{\pi}{4}\right) g_{n}\left(r_{0}, E\right)$.

Using the explicit form of the modes satisfying (13) and (19), we derive the analytic expressions for the induced vacuum current, $j_{\varphi}(r)(15)$, and the induced vacuum magnetic field, $B_{\mathrm{I}}(r)(16)$, in the case of $\nu \geq$ $\geq 1$ and $0<F<1$, and in the case of $\frac{1}{2} \leq \nu<1$ and $\frac{1}{2}\left(\frac{1}{\nu}-1\right)<F<\frac{1}{2}\left(3-\frac{1}{\nu}\right)$. The results can be presented in the form

$$
\begin{aligned}
& j_{\varphi}(r)=j_{\varphi}^{(a)}(r)+j_{\varphi}^{(b)}\left(r ; r_{0}\right), \\
& B_{\mathrm{I}}(r)=B_{\mathrm{I}}^{(a)}(r)+B_{\mathrm{I}}^{(b)}\left(r ; r_{0}\right),
\end{aligned}
$$

where the whole dependence on $r_{0}$ is contained in $j_{\varphi}^{(b)}$ and $B_{\mathrm{I}}^{(b)}$, moreover,

$\lim _{r_{0} \rightarrow 0} j_{\varphi}^{(b)}\left(r ; r_{0}\right)=0, \quad \lim _{r_{0} \rightarrow 0} B_{\mathrm{I}}^{(b)}\left(r ; r_{0}\right)=0$.

The crucial point is the behavior of $j_{\varphi}^{(b)}$ and $B_{\mathrm{I}}^{(b)}$ at $r \rightarrow r_{0}$. If

$\lim _{r \rightarrow r_{0}} j_{\varphi}^{(b)}\left(r ; r_{0}\right)\left(r-r_{0}\right)^{2}=0$

and, consequently,

$\lim _{r \rightarrow r_{0}} B_{\mathrm{I}}^{(b)}\left(r ; r_{0}\right)\left(r-r_{0}\right)=0$,

then the flux $\Phi_{\mathrm{I}}(17)$ is finite. A careful numerical analysis reveals that condition (22) is fulfilled in the cases $\theta=0$ and $\theta=\pi$ only. The case of $F=1 / 2$ needs a special comment, because of the oddness in $\theta$ in this case. Whereas the current and, consequently, 
the induced magnetic field with its flux vanish at $\theta=$ 0 , they are nonvanishing and discontinuous in $\theta$ at $\theta=\pi$. Namely, we obtain

$$
\begin{aligned}
& \left.\Phi_{\mathrm{I}}\right|_{F=1 / 2, \theta=\pi_{ \pm}}= \\
& = \pm \frac{e}{8 m} e^{2 m r_{0}}\left[\Gamma\left(2,2 m r_{0}\right)-4 m^{2} r_{0}^{2} \Gamma\left(0,2 m r_{0}\right)\right]
\end{aligned}
$$

where

$\Gamma(z, u)=\int_{u}^{\infty} d y y^{z-1} \mathrm{e}^{-y}$

is the incomplete gamma-function; in particular,

$\left.\lim _{r_{0} \rightarrow 0} \Phi_{\mathrm{I}}\right|_{F=1 / 2, \theta=\pi_{ \pm}}= \pm \frac{e}{8 m}$.

In the case of $F \neq 1 / 2$, the continuity in $\theta$ is maintained, and we obtain the following representation for the induced vacuum magnetic flux:

$\left.\Phi_{\mathrm{I}}\right|_{\theta=\frac{\pi}{2} \mp \frac{\pi}{2}}=\left.\Phi_{\mathrm{I}}^{(a)}\right|_{\theta=\frac{\pi}{2} \mp \frac{\pi}{2}}+\left.\Phi_{\mathrm{I}}^{(b)}\right|_{\theta=\frac{\pi}{2} \mp \frac{\pi}{2}}, \quad F \neq 1 / 2$,

where

$$
\begin{aligned}
& \left.\Phi_{\mathrm{I}}^{(a)}\right|_{\theta=\frac{\pi}{2} \mp \frac{\pi}{2}}= \\
& =\frac{e}{4 \nu m}\left\{\sum_{p=1}^{[|\nu / 2|]} \exp \left[-2 m r_{0} \sin (p \pi / \nu)\right] \frac{\sin [(2 F-1) p \pi]}{\sin ^{3}(p \pi / \nu)}-\right.
\end{aligned}
$$$$
\left.-\frac{\nu}{4 N}(-1)^{N} \sin (2 N F \pi) \mathrm{e}^{-2 m r_{0}} \delta_{\nu, 2 N}\right\}+
$$$$
+\operatorname{sgn}\left(F-\frac{1}{2}\right) \frac{e}{8 \pi m} \int_{0}^{\infty} \frac{d u}{\cosh ^{3}(u / 2)} \mathrm{e}^{-2 m r_{0} \cosh (u / 2)} \times
$$$$
\times\left\{\cos \left[\nu\left(F-\frac{1}{2}\right) \pi\right)\right] \cosh \left[\nu\left(\left|F-\frac{1}{2}\right|-1\right) u\right]-
$$$$
\left.-\cos \left[\nu\left(\left|F-\frac{1}{2}\right|-1\right) \pi\right] \cosh \left[\nu\left(F-\frac{1}{2}\right) u\right]\right\} \times
$$$$
\times[\cosh (\nu u)-\cos (\nu \pi)]^{-1} \text {, }
$$

$\left.\Phi_{\mathrm{I}}^{(b)}\right|_{\theta=\frac{\pi}{2} \mp \frac{\pi}{2}}=\frac{e}{(4 \pi)^{2}} r_{0} \int_{m r_{0}}^{\infty} \frac{d v v}{\sqrt{v^{2}-m^{2} r_{0}^{2}}} \times$

$\times\left\{\frac{1}{2}\left[\operatorname{sgn}\left(F-\frac{1}{2}\right)\left(C_{\frac{1}{2}+\nu\left(F-\frac{1}{2}\right)}^{( \pm)}(v)+C_{\frac{1}{2}-\nu\left(F-\frac{1}{2}\right)}^{( \pm)}(v)\right)+\right.\right.$

ISSN 2071-0194. Ukr. J. Phys. 2019. Vol. 64, No. 11
$\left.+C_{\frac{1}{2}+\nu\left(F-\frac{1}{2}\right)}^{( \pm)}(v)-C_{\frac{1}{2}-\nu\left(F-\frac{1}{2}\right)}^{( \pm)}(v)\right] D_{\frac{1}{2}+\nu\left|F-\frac{1}{2}\right|}(v)+$

$+\sum_{l=1}^{\infty}\left[C_{\nu\left(l+F-\frac{1}{2}\right)+\frac{1}{2}}^{( \pm)}(v) D_{\nu\left(l+F-\frac{1}{2}\right)+\frac{1}{2}}(v)-\right.$

$\left.\left.-C_{\nu\left(l-F+\frac{1}{2}\right)+\frac{1}{2}}^{( \pm)}(v) D_{\nu\left(l-F+\frac{1}{2}\right)+\frac{1}{2}}(v)\right]\right\}$,

$C_{\rho}^{( \pm)}(v)=\left\{v I_{\rho}(v) K_{\rho}(v) \pm m r_{0}\left[I_{\rho}(v) K_{\rho-1}(v)-\right.\right.$

$\left.\left.-I_{\rho-1}(v) K_{\rho}(v)\right]-v I_{\rho-1}(v) K_{\rho-1}(v)\right\} \times$

$\times\left[v K_{\rho}^{2}(v) \pm 2 m r_{0} K_{\rho}(v) K_{\rho-1}(v)+v K_{\rho-1}^{2}(v)\right]^{-1}$,

and

$D_{\rho}(v)=\rho K_{\rho}^{2}(v)-(\rho-1) K_{\rho+1}(v) K_{\rho-1}(v)+$

$+v\left[K_{\rho}(v) \frac{d}{d \rho} K_{\rho-1}(v)-K_{\rho-1}(v) \frac{d}{d \rho} K_{\rho}(v)\right]$.

In particular,

$\left.\lim _{r_{0} \rightarrow 0} \Phi_{\mathrm{I}}\right|_{\theta=\frac{\pi}{2} \mp \frac{\pi}{2}}=-\frac{e}{6 m}\left[F-\frac{1}{2}-\frac{1}{2} \operatorname{sgn}\left(F-\frac{1}{2}\right)\right] \times$
$\times\left\{\frac{3}{4}-\nu^{2}\left[\frac{1}{4}-\left|F-\frac{1}{2}\right|-F(1-F)\right]\right\}, \quad F \neq 1 / 2$.

The case of massless quantized spinor field is characterized by certain peculiarities. First, there is the invariance under the transformation $\theta \rightarrow \pi-\theta$. Thus, the results are continuous in $\theta$, and their values at $\theta=0$ and $\theta=\pi$ coincide, in particular,

$\left.j_{\varphi}(r)\right|_{F=\frac{1}{2}, \theta=0}=\left.j_{\varphi}(r)\right|_{F=\frac{1}{2}, \theta=\pi}=0$

and

$\left.B_{\mathrm{I}}(r)\right|_{F=\frac{1}{2}, \theta=0}=\left.B_{\mathrm{I}}(r)\right|_{F=\frac{1}{2}, \theta=\pi}=0$.

Second, instead of the exponential decrease, $j_{\varphi}$ and $B_{\mathrm{I}}$ decrease as $r^{-1}$ at large distances from the ANO vortex. Consequently, the flux $\Phi_{\mathrm{I}}$, see (17), is given by an integral which is linearly divergent at $r \rightarrow \infty$. Therefore, we have no choice but to introduce a cutoff $r_{\max }>r$ and the restricted flux,

$\Phi_{\mathrm{I}\left(r_{\max }\right)}=\frac{2 \pi}{\nu} \int_{r_{0}}^{r_{\max }} d r r B_{\mathrm{I}}(r)$ 
As follows from our numerical analysis of the integrand in (34) near the lower limit of integration, relation (22) is fulfilled, and the flux $\Phi_{\mathrm{I}\left(r_{\max }\right)}(34)$ is finite at $\theta=\frac{\pi}{2} \mp \frac{\pi}{2}$ only. We get immediately:

$\left.\Phi_{\mathrm{I}\left(r_{\max }\right)}\right|_{\theta=\frac{\pi}{2} \mp \frac{\pi}{2}}=0, \quad F=1 / 2$.

As to $F \neq 1 / 2$, although we obtain the analytic expression for $\left.\Phi_{\mathrm{I}\left(r_{\max }\right)}\right|_{\theta=\frac{\pi}{2} \mp \frac{\pi}{2}}$ for arbitrary $r_{\max }>r_{0}$, the physically sensible case is that of $r_{\max } \gg r_{0}$. Retaining only terms which are maximal in the latter case, we get the expression for the flux:

$$
\begin{aligned}
& \left.\Phi_{\mathrm{I}\left(r_{\max }\right)}\right|_{\theta=\frac{\pi}{2} \mp \frac{\pi}{2}}=\frac{e r_{\max }}{4 \nu}\left\{\sum_{p=1}^{[|\nu / 2|]} \frac{\sin [(2 F-1) p \pi]}{\sin ^{2}(p \pi / \nu)}-\right. \\
& \left.-\frac{\nu}{4 N}(-1)^{N} \sin (2 N F \pi) \delta_{\nu, 2 N}\right\}+ \\
& +\operatorname{sgn}\left(F-\frac{1}{2}\right) \frac{e r_{\max }}{8 \pi} \int_{0}^{\infty} \frac{d u}{\cosh ^{2}(u / 2)} \times \\
& \times\left\{\cos \left[\nu\left(F-\frac{1}{2}\right) \pi\right] \cosh \left[\nu\left(\left|F-\frac{1}{2}\right|-1\right) u\right]-\right. \\
& \left.-\cos \left[\nu\left(\left|F-\frac{1}{2}\right|-1\right) \pi\right] \cosh \left[\nu\left(F-\frac{1}{2}\right) u\right]\right\} \times \\
& \times[\cosh (\nu u)-\cos (\nu \pi)]^{-1}+O\left(e r_{0}\right), \quad F \neq 1 / 2,
\end{aligned}
$$

and the relation between the current and the magnetic field:

$$
\begin{aligned}
& \text { עe }\left.j_{\varphi}(r)\right|_{\theta=\frac{\pi}{2} \mp \frac{\pi}{2}}=\left.\frac{r_{\max }}{r_{\max }-r} B_{\mathrm{I}}(r)\right|_{\theta=\frac{\pi}{2} \mp \frac{\pi}{2}}= \\
& =\left.\frac{\nu}{\pi r_{\max } r} \Phi_{\mathrm{I}\left(r_{\max }\right)}\right|_{\theta=\frac{\pi}{2} \mp \frac{\pi}{2}}, \quad r \gg r_{0} .
\end{aligned}
$$

In particular, we get, in the case of $\nu=1$,

$$
\begin{aligned}
& \left.\Phi_{\mathrm{I}\left(r_{\max }\right)}\right|_{\nu=1, \theta=\frac{\pi}{2} \mp \frac{\pi}{2}}= \\
& =\frac{e}{4} r_{\max } \tan (F \pi)\left|F-\frac{1}{2}\right|\left(\left|F-\frac{1}{2}\right|-1\right)+O\left(e r_{0}\right)
\end{aligned}
$$

and

$$
\begin{aligned}
& \left.e j_{\varphi}(r)\right|_{\nu=1, \theta=\frac{\pi}{2} \mp \frac{\pi}{2}}= \\
& =\left.\frac{r_{\max }}{r_{\max }-r} B_{I}(r)\right|_{\nu=1, \theta=\frac{\pi}{2} \mp \frac{\pi}{2}}=
\end{aligned}
$$

$=\frac{e}{4 \pi r} \tan (F \pi)\left|F-\frac{1}{2}\right|\left(\left|F-\frac{1}{2}\right|-1\right), \quad r \gg r_{0}$.

The last relation for the current was first obtained in [24] [see (10.6) in this reference, where the definition of the current differs by extra $\left.r^{-1}\right]$. Note a discontinuity at $F=1 / 2$, which is independent of $\nu$,

$\left.\lim _{F \rightarrow(1 / 2)_{ \pm}} e j_{\varphi}(r)\right|_{\theta \neq \pm \frac{\pi}{2}}= \pm \frac{e}{4 \pi^{2} r}, \quad r \gg r_{0}$.

This is distinct from the case of quantized scalar field under the Dirichlet boundary condition, when the current is continuous and vanishing at $F=1 / 2$ $[44,46,47]$, see the appropriate expression from these references at $m=0$ and $\nu=1$ :

$\left.e j_{\varphi}(r)\right|_{\text {scalar, Dirichlet }}=-\frac{e}{4 \pi r} \tan (F \pi)\left(F-\frac{1}{2}\right)^{2}$.

\section{Discussion and Conclusion}

The effects of conicity, which are characterized by the value of the deficit angle, $8 \pi \mathrm{G} M$, are negligible for the ANO vortices in ordinary superconductors, since the constant $\mathrm{G}$ is of order of the Planck length squared, and the quantity $M$ is of order of the inverse correlation length squared. However, topological defects of the type of ANO vortices also arize in another field in cosmology and high energy physics, where they attained the name of cosmic strings $[48,49]$. Cosmic strings with $8 \pi$ G $M \sim 1$ are definitely ruled out by astrophysical observations, but there remains a room for cosmic strings with $8 \pi \mathrm{G} M \sim 10^{-6}$ and less (see, e.g., [50]), although the direct evidence for their existence is lacking.

A recent development in material science also provides an unexpected link between condensed matter and high-energy physics, which is caused to a large extent by the experimental discovery of graphene - a two-dimensional crystalline allotrope formed by a monolayer of carbon atoms [51]. A single topological defect (disclination) warps a sheet of graphene, rolling it into a nanocone which is similar to the transverse section of a spatial region out of a cosmic string; carbon nanocones with deficit angles equal to $N_{d} \pi / 3\left(N_{d}\right.$ is a possible number of sectors which are removed from the hexagonal lattice: $N_{d}=1,2,3,4,5$, i.e. $\left.\nu=\frac{6}{5}, \frac{3}{2}, 2,3,6\right)$ were observed experimentally, see [52] and references therein. Moreover, theory also predicts saddlelike nanocones with the deficit angle taking negative values unbounded from below (sectors can be

ISSN 2071-0194. Ukr. J. Phys. 2019. Vol. 64, No. 11 
added: $N_{d}=-1,-2,-3,-4,-5,-6, \ldots,-\infty$, i.e. $\nu=$ $\left.=\frac{6}{7}, \frac{3}{4}, \frac{2}{3}, \frac{3}{5}, \frac{6}{11}, \frac{1}{2}, \ldots, 0\right)$, which can be regarded as corresponding to the cosmic strings with negative mass density. Note that nanoconical structures may arise as well in a diverse set of condensed matter systems known as the two-dimensional Dirac materials, ranging from honeycomb crystalline allotropes (silicene and germanene [53], phosphorene [54]) to hightemperature cuprate superconductors [55] and topological insulators [56].

Since the transverse size of the ANO vortex is related to the correlation length, its nonvanishing value, $r_{0}$, should be taken into account. We have considered the current and the magnetic field which are induced in the vacuum of a quantized spinor field in the case of $\nu \geq 1$ and $0<F<1$, and in the case of $\frac{1}{2} \leq \nu<1$ and $\frac{1}{2}\left(\frac{1}{\nu}-1\right)<$ $<F<\frac{1}{2}\left(3-\frac{1}{\nu}\right)$. The dependence of these characteristics of the vacuum on boundary conditions ensuring the impenetrability of the vortex core is analyzed, and we find that the demand of finiteness of the total induced vacuum magnetic flux removes an ambiguity in the choice of boundary conditions. The case of massless quantized spinor field requires the introduction of the maximal size of a system, $r_{\max }$. We discover that, for physically sensible values $r_{\max } \gg r_{0}$, the vacuum polarization effects in this case, in distinction from the case of massive quantized spinor field, are independent of the transverse size of the ANO vortex. Due to this distinction, the results in the massless case are discontinuous at $F=1 / 2$ with a jump which is independent of $\nu$, whereas the results in the massive case at $\theta=0$ are continuous in $F$ and vanishing at $F=1 / 2$, as long as the transverse size of the vortex is nonvanishing.

The work was presented at the XI International Bolyai-Gauss-Lobachevskii (BGL-2019) Conference on Non-Euclidean, Non-Commutative Geometry and Quantum Physics, May 19-24, Kyiv, Ukraine. Yu.A.S. acknowledges a support from the National Academy of Sciences of Ukraine (project No.01172U000237), from the Program of Fundamental Research of the Department of Physics and Astronomy of the National Academy of Sciences of Ukraine (project No.0117U000240), and from the ICTP - SEENET-MTP project NT-03 "Cosmology Classical and Quantum Challenges".

ISSN 2071-0194. Ukr. J. Phys. 2019. Vol. 64, No. 11
1. Y. Aharonov, D. Bohm. Significance of electromagnetic potentials in the quantum theory. Phys. Rev. 115, 485 (1959).

2. M. Peshkin, A. Tonomura. The Aharonov-Bohm Effect (Springer, 1989).

3. I.V. Krive, A.S. Rozhavsky. Non-traditional AharonovBohm effects in condensed matter. Int. J. Mod. Phys. B 6, 1255 (1992).

4. A. Tonomura. The AB effect and its expanding applications. J. Phys. A: Math. Theor. 43, (2010) 354021.

5. A.A. Abrikosov. On the magnetic properties of superconductors of the second group. Sov. Phys. - JETP 5, 1174 (1957).

6. H.B. Nielsen, P. Olesen. Vortex-line models for dual strings. Nucl. Phys. B 61, 45 (1973).

7. R.P. Huebener. Magnetic Flux Structure in Superconductors (Springer, 1979).

8. M. Reed, B. Simon. Methods of Modern Mathematical Physics II. Fourier Analysis, Self-Adjointness (Academic Press, 1975).

9. D. Garfinkle. General relativistic strings. Phys. Rev. D 32, 1323 (1985)

10. C.W. Misner, K.S. Thorne, J.A. Wheeler. Gravitation (W.H. Freeman, 1973).

11. G.V. Dunne. Topological Aspects of Low Dimensional Systems (Springer, 1999).

12. E.C. Marino. Quantum Field Theory Approach to Condensed Matter Physics (Cambridge Univ. Press, 2017).

13. A.J. Niemi, G.W. Semenoff. Fermion number fractionization in quantum field theory. Phys. Rep. 135, 99 (1984).

14. Yu.A. Sitenko. On the electron charge fractionization in magnetic field with boundaries present. Sov. J. Nucl. Phys. 47, 184 (1988).

15. Yu.A. Sitenko. Electron-charge fractionization on surfaces of various geometry in an external magnetic field. Nucl. Phys. B 342, 655 (1990).

16. Yu.A. Sitenko. Geometry of the base manifold and fermion number fractionization. Phys. Lett. B 253, 138 (1991).

17. Yu.A. Sitenko. Self-adjointness of the Dirac Hamiltonian and fermion number fractionization in the background of a singular magnetic vortex. Phys. Lett. B 387, 334 (1996).

18. Yu.A. Sitenko. Self-adjointness of the Dirac Hamiltonian and vacuum quantum numbers induced by a singular external field. Phys. Atom. Nucl. 60, 2102 (1997); (E) 62, 1084 (1999).

19. Yu.A. Sitenko, D.G. Rakityansky. Quantum numbers of the theta vacuum in $(2+1)$-dimensional spinor electrodynamics: Charge and magnetic flux. Phys. Atom. Nucl. 60, 1497 (1997).

20. Yu.A. Sitenko. Effects of fermion vacuum polarization by a singular magnetic vortex: Zeta function and energy. Phys. Atom. Nucl. 62, 1056 (1999). 
21. Yu.A. Sitenko. Polarization of a fermion vacuum by a singular magnetic vortex: Spin and angular momentum. Phys. Atom. Nucl. 62, 1767 (1999).

22. Yu.A. Sitenko. Induced vacuum condensates in the background of a singular magnetic vortex in $2+1$-dimensional space-time. Phys. Rev. D 60, 125017 (1999).

23. Yu.A. Sitenko. Chiral symmetry breaking as a consequence of nontrivial spatial topology. Mod. Phys. Lett. A 14, 701 (1999).

24. Yu.A. Sitenko. Self-adjointness of the two-dimensional massless Dirac Hamiltonian and vacuum polarization effects in the background of a singular magnetic vortex. Annals Phys. 282, 167 (2000).

25. P. Gornicki. Aharonov-Bohm effect and vacuum polarization. Annals Phys. 202, 271 (1990).

26. E.G. Flekkoy, J.M. Leinaas. Vacuum currents around a magnetic flux string. Int. J. Mod. Phys. A 6, 5327 (1991).

27. R.R. Parwani, A.S. Goldhaber. Decoupling in $(2+1)$-dimensional QED? Nucl. Phys. B 359, 483 (1991).

28. V.P. Frolov, E.M. Serebriany. Vacuum polarization in the gravitational field of a cosmic string. Phys. Rev. D 35, 3779 (1987).

29. J.S. Dowker. Vacuum averages for arbitrary spin around a cosmic string. Phys. Rev. D 36, 3742 (1987).

30. M.E.X. Guimaraes, B. Linet. Scalar Green's functions in an Euclidean space with a conical-type line singularity. Commun. Math. Phys. 165, 297 (1994).

31. E.S. Moreira. Massive quantum fields in a conical background. Nucl. Phys. B 451, 365 (1995).

32. M. Bordag, K. Kirsten, S. Dowker. Heat-kernels and functional determinants on the generalized cone. Commun. Math. Phys. 182, 371 (1996).

33. D. Iellici. Massive scalar field near a cosmic string. Class. Quantum Grav. 14, 3287 (1997).

34. L. Sriramkumar. Fluctuations in the current and energy densities around a magnetic flux carrying cosmic string. Class. Quantum Grav. 18, 1015 (2001).

35. J. Spinelly, E.R. Bezerra de Mello. Spinor Green function in higher-dimensional cosmic string space-time in the presence of magnetic flux. J. High Energy Phys. 09, 005 (2008).

36. E.R. Bezerra de Mello, V. Bezerra, A.A. Saharian, V.M. Bardeghyan. Fermionic current densities induced by magnetic flux in a conical space with a circular boundary. Phys. Rev. D 82, 085033 (2010).

37. S. Bellucci, E.R. Bezerra de Mello, A.A. Saharian. Fermionic condensate in a conical space with a circular boundary and magnetic flux. Phys. Rev. D 83, 085017 (2011).

38. E.R. Bezerra de Mello, F. Moraes, A.A. Saharian. Fermionic Casimir densities in a conical space with a circular boundary and magnetic flux. Phys. Rev. D 85, 045016 (2012).
39. V.M. Gorkavenko, Yu.A. Sitenko, O.B. Stepanov. Polarization of the vacuum of a quantized scalar field by an impenetrable magnetic vortex of finite thickness. J. Phys. A: Math. Theor. 43, 175401 (2010).

40. V.M. Gorkavenko, Yu.A. Sitenko, O.B. Stepanov. Vacuum energy induced by an impenetrable flux tube of finite radius. Int. J. Mod. Phys. A 26, 3889 (2011).

41. V.M. Gorkavenko, Yu.A. Sitenko, O.B. Stepanov. Casimir energy and force induced by an impenetrable flux tube of finite radius. Int. J. Mod. Phys. A 28, 1350161 (2013).

42. V.M. Gorkavenko, I.V. Ivanchenko, Yu.A. Sitenko. Induced vacuum current and magnetic field in the background of a vortex. Int. J. Mod. Phys. A 31, 1650017 (2016).

43. Yu.A. Sitenko, V.M. Gorkavenko. Induced vacuum energymomentum tensor in the background of a $(\mathrm{d}-2)$-brane in $(\mathrm{d}+1)$-dimensional space-time. Phys. Rev. D 67, 085015 (2003).

44. Yu.A. Sitenko, N.D. Vlasii. Induced vacuum current and magnetic field in the background of a cosmic string. Class. Quantum Grav. 26, 195009 (2009).

45. Yu.A. Sitenko, N.D. Vlasii. Induced vacuum energymomentum tensor in the background of a cosmic string. Class. Quantum Grav. 29, 095002 (2012).

46. Yu.A. Sitenko, A.Yu. Babansky. The Casimir-AharonovBohm effect? Mod. Phys. Lett. A 13, 379 (1998).

47. Yu.A. Sitenko, A.Yu. Babansky. Effects of boson-vacuum polarization by a singular magnetic vortex. Phys. At. Nucl. 61, 1594 (1998).

48. A. Vilenkin, E.P.S. Shellard. Cosmic Strings and Other Topological Defects (Cambridge Univ. Press, 1994).

49. M.B. Hindmarsh, T.W.B. Kibble. Cosmic strings. Rep. Prog. Phys. 58, 477 (1995).

50. R.A. Battye, B. Garbrecht, A. Moss, H. Stoica. Constraints on brane inflation and cosmic strings. J. Cosmol. Astropart. Phys. JCAP 0801, 020 (2008).

51. A.K. Geim, K.S. Novoselov. The rise of graphene. Nature Mater. 6, 183 (2007).

52. S.N. Naess, A. Elgsaeetter, G. Helgesen, K.D. Knudsen. Carbon nanocones: Wall structure and morphology. Sci. Technol. Adv. Mat. 10, 065002 (2009).

53. S. Cahangirov, M. Topsakal, E. Akturk, H. Sahin, S. Ciraci. Two- and one-dimensional honeycomb structures of silicon and germanium. Phys. Rev. Lett. 102, 236804 (2009).

54. H. Liu, A.T. Neal, Z. Zhu, Z. Luo, X. Xu, D. Tomanek, P.D. Ye. Phosphorene: an unexplored 2D semiconductor with a high hole mobility. ACS NANO 8, 4033 (2014).

55. C.C. Tsuei, J.R. Kirtley. Pairing symmetry in cuprate superconductors. Rev. Mod. Phys. 72, 969 (2000).

56. X.L. Qi, S.C. Zhang. Topological insulators and superconductors. Rev. Mod. Phys. 83, 1057 (2011).

Received 22.01.09

ISSN 2071-0194. Ukr. J. Phys. 2019. Vol. 64, No. 11 
Ю.А. Ситенко, В.М. Горкавенко

ПОЛЯРИЗАЦІЯ ВАКУУМУ

КВАНТОВАНОГО СПІНОРНОГО ПОЛЯ

ЗА НАЯВНОСТІ ТОПОЛОГІЧНОГО ДЕФЕКТУ

У ДВОВИМІРНОМУ ПРОСТОРІ

$\mathrm{P}$ е $з$ ю м е

Двовимірний простір з топологічним дефектом $є$ поперечним зрізом тривимірного простору з вихором АбрикосоваНільсена-Олесена, який являє собою непроникливу для квантованої матерії трубку з потоком калібрувального поля. Заряджене поле спінорної матерії квантується в цьому зрізі, задовольняючи найбільш загальним математично допустимим граничним умовам. Показано, що струм та магнітне поле індукуються у вакуумі. Вивчається залежність результатів від граничних умов. Встановлено, що вимога скінченності повного індукованого вакуумного магнітного потоку усуває неоднозначність у виборі граничних умов. Обговорюються відмінності між випадками масивної та безмасової спінорної матерії. 